\title{
On Maps which Preserve Equality of Distance in $F^{*}$-spaces
}

by

\section{Dongni TAN}

\author{
Presented by Aleksander PEŁCZYŃSKI
}

Summary. We prove that every map $T$ between two $F^{*}$-spaces which preserves equality of distance and satisfies $T(0)=0$ is linear.

1. Preliminaries and introduction. Recall from [7] that a non-negative function $\|\cdot\|$ defined on a linear space $E$ is called an $F$-norm provided

(i) $\|x\|=0$ iff $x=0$;

(ii) $\|a x\|=\|x\|$ for all $a$ with $|a|=1$;

(iii) $\|x+y\| \leqslant\|x\|+\|y\|$;

(iv) $\left\|a_{n} x\right\| \rightarrow 0$ provided $a_{n} \rightarrow 0$;

(v) $\left\|a x_{n}\right\| \rightarrow 0$ provided $x_{n} \rightarrow 0$.

An $F$-norm $\|\cdot\|$ induces a translation invariant distance $d$ by

$$
d(x, y)=\|x-y\| \quad \text { for all } x, y \in E .
$$

A linear space $E$ with an $F$-norm $\|\cdot\|$ is said to be an $F^{*}$-space. A complete $F^{*}$-space is called an $F$-space.

Let $E=(E,\|\cdot\|), F=(F,\|\cdot\|)$ be two $F^{*}$-spaces and $\mathbb{R}_{0}^{+}=\{t \in \mathbb{R}$ : $0 \leq t<\infty\}$. We say that a map $T: E \rightarrow F$ preserves equality of distance if there exists a function $\phi: \mathbb{R}_{0}^{+} \rightarrow \mathbb{R}_{0}^{+}$such that for all $x, y \in E$,

$$
\|T x-T y\|=\phi(\|x-y\|) .
$$

2000 Mathematics Subject Classification: Primary 46A99.

Key words and phrases: $F^{*}$-space, equality of distance preserving map, isometry.

This paper is supported by The National Natural Science Foundation of China (10571090) and the Research Fund for the Doctoral Program of Higher Education (20080055010). 
The function $\phi$ is called the gauge function for $T$, and this definition equivalently means that

$$
u, v, z, w \in E,\|u-v\|=\|z-w\| \Rightarrow\|T u-T v\|=\|T z-T w\| .
$$

In particular, when $\phi$ is the identity function, the map $T$ is an isometry. Such mappings were studied by Schoenberg [9] and by von Neumann and Schoenberg [5] for Hilbert spaces.

The classical theorem of Mazur-Ulam [4] states that an onto isometry between two real normed spaces is affine. Charzyński [2] and Rolewicz [8] have shown, respectively, that surjective isometries of finite-dimensional $F$ spaces and of locally bounded spaces with concave norm are also linear. Ding and Huang [3] have extended the result of Mazur-Ulam to locally midpoint constricted $F$-spaces. More generally, Vogt [10] has shown that continuous surjective equality of distance preserving maps between two real normed spaces are affine.

The present paper extends the result of Vogt to a large class of $F^{*}$-spaces including all $p$-normed spaces $(0<p \leq 1)$. The proof of the main result here depends on the technique of Vogt. All the linear spaces mentioned in this paper are assumed to be real.

2. Main results. The following lemma in metric space theory was proved by Vogt [10]; it is similar to one stated by Aronszajn [1].

Lemma 2.1. Let $(M, d)$ be a bounded metric space. Suppose there exists an element $m$ in $M$, a surjective isometry $V: M \rightarrow M$, and a constant $K>1$ such that $d(V x, x) \geq K d(m, x)$ for all $x$ in $M$. Then $m$ is a fixed point for every surjective isometry $S: M \rightarrow M$.

Rassias [6] shows that the ratio $\|2 x\| /\|x\|$ plays an important role in generalizations of the Mazur-Ulam theorem. It is also of importance in the following statement.

Theorem 2.2. Let $(E,\|\cdot\|),(F,\|\cdot\|)$ be two real $F^{*}$-spaces. Suppose that there exists a number $r>0$ such that

$$
\alpha_{F}(r)=\inf \{\|2 x\| /\|x\|: x \in F, 0<\|2 x\| \leq r\}>1 .
$$

Let $T: E \rightarrow F$ with $T(0)=0$ be a continuous surjective map which preserves equality of distance, i.e. there exists a function $\phi: \mathbb{R}_{0}^{+} \rightarrow \mathbb{R}_{0}^{+}$such that for all $x, y$ in $E$,

$$
\|T x-T y\|=\phi(\|x-y\|) .
$$

Then $T$ is linear.

Proof. Since $T$ is continuous, there is a $\delta>0$ such that for any $a, b \in E$ satisfying $\|(a-b) / 2\| \leq \delta$, we have $\|T((a-b) / 2)\| \leq r / 4$. 
Step 1. Fix any $a, b \in E$ satisfying $\|(a-b) / 2\| \leq \delta$. Let $m=T\left(\frac{a-b}{2}\right) \quad$ and $\quad M:=\{y \in F:\|y\|=\|2 m-y\| \leq 2\|m\| \leq r / 2\}$.

Define

$$
V: M \rightarrow M \quad \text { by } \quad V(y)=2 m-y .
$$

It follows that

(i) $M \neq \emptyset$ with $m \in M$.

(ii) $M$ is a bounded metric space.

(iii) $V$ is an isometry from $M$ onto $M$ since $V^{2}=\operatorname{id}_{M}$ and $\| V\left(y_{1}\right)-$ $V\left(y_{2}\right)\|=\| 2 m-y_{1}-2 m+y_{2}\|=\| y_{1}-y_{2} \|$ for all $y_{1}, y_{2} \in M$.

(iv) $d(V y, y)=\|V y-y\|=\|2(m-y)\| \geq K\|m-y\|=K d(m, y)$ with $K=\alpha_{F}(r)>1$.

Hence the conditions of Lemma 2.1 are satisfied. Thus $m=T((a-b) / 2)$ is a fixed point for every surjective isometry of $M$.

Let $x_{0} \in E$ be such that

$$
T\left(x_{0}\right)=2 T\left(\frac{a-b}{2}\right)=2 m .
$$

Define $S: M \rightarrow M$ by $S(y)=T\left(x_{0}-T^{-1}(y)\right)$. We shall prove that $S$ is well-defined and it is an isometry from $M$ onto $M$.

- $S$ is well-defined. Indeed, if $T\left(x_{1}\right)=T\left(x_{2}\right)=y$, then

$$
\left\|T\left(x_{0}-x_{1}\right)-T\left(x_{0}-x_{2}\right)\right\|=\phi\left(\left\|x_{1}-x_{2}\right\|\right)=\left\|T x_{1}-T x_{2}\right\|=0 .
$$

- $S$ is an isometry from $M$ onto $M$. For any $y_{1}, y_{2} \in M$ with $T\left(x_{1}\right)=y_{1}$ and $T\left(x_{2}\right)=y_{2}$, by the definition of $S$, we get

$$
\begin{aligned}
\left\|S\left(y_{1}\right)-S\left(y_{2}\right)\right\| & =\left\|T\left(x_{0}-x_{1}\right)-T\left(x_{0}-x_{2}\right)\right\| \\
& =\phi\left(\left\|x_{1}-x_{2}\right\|\right)=\left\|T x_{1}-T x_{2}\right\|=\left\|y_{1}-y_{2}\right\| .
\end{aligned}
$$

Moreover for every $y \in M$ with $T(x)=y$, we have

$$
S(y)=T\left(x_{0}-x\right) \text { and } \quad\|T x\|=\|2 m-T x\| .
$$

By (2) and (3) and the fact that $T(0)=0$, it is easy to see that

$$
\begin{aligned}
\|S y\| & =\left\|T\left(x_{0}-x\right)\right\|=\left\|T\left(x_{0}-x\right)-T(0)\right\| \\
& =\phi\left(\left\|x_{0}-x\right\|\right)=\left\|T x_{0}-T x\right\| \\
& =\|2 m-T x\|=\|T x\|=\|T(x)-T(0)\| \\
& =\phi(\|x-0\|)=\phi\left(\left\|x_{0}-\left(x_{0}-x\right)\right\|\right) \\
& =\left\|T\left(x_{0}\right)-T\left(x_{0}-x\right)\right\|=\|2 m-S y\| .
\end{aligned}
$$

This implies $S(M) \subseteq M$ and by the definition of $S$, we can easily get $S^{2}=\operatorname{id}_{M}$, hence $S(M)=M$. 
It now follows from Lemma 2.1 that $m$ is a fixed point of $S$, i.e.,

$$
T\left(\frac{a-b}{2}\right)=m=S m=T\left(x_{0}-\frac{a-b}{2}\right) .
$$

We apply equations (2) and (4) to get

$$
\begin{aligned}
\left\|T(a-b)-2 T\left(\frac{a-b}{2}\right)\right\| & =\left\|T(a-b)-T x_{0}\right\|=\phi\left(\left\|a-b-x_{0}\right\|\right) \\
& =\phi\left(\left\|\frac{a-b}{2}-\left(x_{0}-\frac{a-b}{2}\right)\right\|\right) \\
& =\left\|T\left(\frac{a-b}{2}\right)-T\left(x_{0}-\frac{a-b}{2}\right)\right\|=0,
\end{aligned}
$$

that is,

$$
T(a-b)=2 T\left(\frac{a-b}{2}\right) .
$$

For fixed $b$, define $T_{b}: E \rightarrow F$ by $T_{b}(x)=T(x+b)-T(b)$. Then $T_{b}$ has the following properties:

(i) $T_{b}$ is a continuous map which preserves equality of distance with $T_{b}(0)=0$ and it has the same gauge function $\phi$ as $T$.

This follows from

$$
\begin{gathered}
\left\|T_{b}(x)-T_{b}(y)\right\|=\|T(x+b)-T(b)-T(y+b)+T(b)\| \\
=\|T(x+b)-T(y+b)\|=\phi(\|x-y\|) . \\
\text { (ii) }\left\|T_{b}\left(\frac{a-b}{2}\right)\right\|=\left\|T_{b}\left(\frac{a-b}{2}\right)-T_{b}(0)\right\|=\phi\left(\left\|\frac{a-b}{2}\right\|\right)=\left\|T\left(\frac{a-b}{2}\right)\right\| \leq \frac{r}{4} .
\end{gathered}
$$

Properties (i) and (ii) show that $T_{b}$ has the same properties as $T$. Therefore we conclude that

$$
T_{b}(a-b)=2 T_{b}\left(\frac{a-b}{2}\right) .
$$

It follows that

$$
\begin{aligned}
T\left(\frac{a+b}{2}\right) & =T\left(\frac{a-b}{2}+b\right)-T(b)+T(b) \\
& =T_{b}\left(\frac{a-b}{2}\right)+T(b)=\frac{T_{b}(a-b)}{2}+T(b) \\
& =\frac{T(a-b+b)-T(b)}{2}+T(b)=\frac{T(a)+T(b)}{2}
\end{aligned}
$$

for all $a, b \in E$ with $\|(a-b) / 2\| \leq \delta$. 
STEP 2. In case $\|(a-b) / 2\|>\delta$, we may also show by induction and Step 1 that

$$
T\left(\frac{a+b}{2}\right)=\frac{T(a)+T(b)}{2} .
$$

We can find a detailed proof for Step 2 in [7] and also in [8].

From Steps 1 and 2, for every $a, b \in E$ we obtain

$$
T(a)=T\left(\frac{2 a+0}{2}\right)=\frac{T(2 a)+T(0)}{2}=\frac{T(2 a)}{2}
$$

and

$$
T(a+b)=T\left(\frac{2 a+2 b}{2}\right)=\frac{T(2 a)+T(2 b)}{2}=T(a)+T(b) .
$$

Hence $T$ is additive. Since it is continuous and the spaces are real, it is easy to check that it is a linear operator.

Corollary 2.3. Let $E, F$ be p-normed spaces $(0<p \leq 1)$, and let $T: E \rightarrow F$ with $T(0)=0$ be a surjective continuous map which preserves equality of distance. Then

(i) $T$ is linear,

(ii) $T=r V$ where $r \in \mathbb{R}$ and $V$ is an isometry.

Proof. It is easy to see that (i) is a consequence of Theorem 2.1 and we only need to prove (ii). Let $\phi$ be the gauge function for $T$. Since $T$ is linear, we see that for all $x \in E$ with $x \neq 0$,

$$
\frac{\|T x\|}{\|x\|}=\left\|T\left(\frac{x}{\|x\|^{1 / p}}\right)\right\|=\phi(1) .
$$

Obviously, the equation $\|T x\|=\phi(1)\|x\|$ holds for $x=0$. Thus the proof is complete.

When $\phi$ is the identity function, i.e., $\phi(t)=t$ for all $t \geq 0$, from Theorem 2.2 we get the following result for isometries between $F^{*}$-spaces.

Corollary 2.4. Let E, $F$ be $F^{*}$-spaces. Suppose that there exists a positive number $r$ such that $\inf \{\|2 x\| /\|x\|: x \in F, 0<\|2 x\| \leq r\}>1$. Let $V: E \rightarrow F$ with $V(0)=0$ be a surjective isometry, i.e.

$$
\|V x-V y\|=\|x-y\| \quad \text { for all } x, y \in E .
$$

Then $V$ is linear.

REMARK. Rolewicz's book [7, p. 397] and his paper [8] contain the following result: Let a map $T$ from a locally bounded space onto another locally bounded space be an isometry with $T(0)=0$. If the norms on two spaces are concave, then $T$ is linear. It should be pointed out that Rolewicz's proof of this result can also be applied to proving the above corollary. 
Acknowledgments. I would like to express my sincere gratitude to Professor Ding Guanggui for his encouraging advice and the referee for his helpful suggestions that improved this paper.

\section{References}

[1] N. Aronszajn, Caractérisation métrique de l'espace de Hilbert, des espaces vectoriels et de certains groupes métriques, C. R. Acad. Sci. Paris 201 (1935), 811-813.

[2] Z. Charzyński, Sur les transformations isométriques des espaces du type F, Studia Math. 13 (1953), 94-121.

[3] G. G. Ding and S. Z. Huang, On extension of isometries in (F)-space, Acta Math. Sin. (Engl. Ser.) 121 (1996), 1-9.

[4] S. Mazur et S. Ulam, Sur les transformations isométriques d'espaces vectoriels normés, C. R. Acad. Sci. Paris 194 (1932), 946-948.

[5] J. von Neumann and I. J. Schoenberg, Fourier integrals and metric geometry, Trans. Amer. Math. Soc. 50 (1941), 226-251.

[6] Th. M. Rassias, Properties of isometric mappings, J. Math. Anal. Appl. 235 (1999), $108-121$.

[7] S. Rolewicz, Metric Linear Spaces, 2nd ed., Reidel and PWN-Polish Sci. Publ., Dordrecht-Warszawa, 1985.

[8] —, A generalization of the Mazur-Ulam theorem, Studia Math. 31 (1968), 501-505.

[9] I. J. Schoenberg, Metric spaces and completely monotone functions, Ann. of Math. 39 (1938), 811-841.

[10] A. Vogt, Maps which preserve equality of distance, Studia Math. 45 (1973), 43-48.

Dongni Tan

School of Mathematical Sciences

Nankai University

Tianjin 300071, China

E-mail: 0110127@mail.nankai.edu.cn

Received May 4, 2008;

received in final form October 10, 2008 